NASA Technical Memorandum 102846

\title{
Sideslip-Induced Static Pressure Errors in Flight-Test Measurements
}

Edwin K. Parks, University of Arizona, Tucson, Arizona

Ralph E. Bach, Jr., Ames Research Center, Moffett Field, California

Duc Tran, San Jose State University, San Jose, California

June 1990

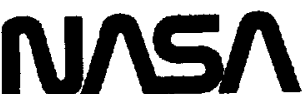

National Aeronautics and

Space Administration 


$$
\longrightarrow
$$




\section{SIDESLIP-INDUCED STATIC PRESSURE ERRORS IN FLIGHT-TEST MEASUREMENTS}

by

Edwin K. Parks"

University of Arizona

Tucson, AZ 85721

\author{
Ralph E. Bach, Jr."* \\ NASA Ames Research Center \\ Moffett Field, CA 94035
}

\author{
Duc Tran ${ }^{\dagger}$ \\ San Jose State University \\ San Jose, CA 95192
}

\section{Abstract}

During lateral flight-test maneuvers of a V/STOL research aircraft, large errors in static pressure were observed. An investigation of the data showed a strong correlation of the pressure record with variations in sideslip angle. The sensors for both measurements were located on a standard air-data nose boom. This paper describes an algorithm based on potential flow over a cylinder that was developed to correct the pressure record for sideslip-induced errors. In order to properly apply the correction algorithm, it was necessary to estimate and correct the lag error in the pressure system. The method developed for estimating pressure lag is based on the coupling of sideslip activity into the static ports and can be used as a standard flight-test procedure. The paper discusses the estimation procedure and presents the corrected static-pressure record for a typical lateral maneuver. It is shown that application of the correction algorithm effectively attenuates sideslip-induced errors.

\section{Introduction}

In the flight-testing of aircraft, it is essential that accurate measurements of static and total air pressure be obtained. Usually, the pressure sensors are mounted ahead of the aircraft, near the tip of a nose boom, to better sense the "free stream" conditions. However, unless a swivel pitot static head is used, the pressure measurements will be in error whenever the boom is yawed with respect to the direction of airflow. The static-pressure measurement is the most affected: its errors depend on the angles of attack and sideslip of the boom as well as on the number and location of static ports around the circumference of the boom. Gracey discusses this effect as well as other

\footnotetext{
- Profeseor of Aerospace Engineering, Associate Fellow, AIAA - Aerospece Engineer, Member, AIAA

t Graduate student, Dept. of Mechanical Engineering

Copyright 1990 by the American Institute of Aeronautics and Astronautics, Inc. No copyright is asserted in the United States under Title 17, U.S. Code. The U.S. Govemment has a royaltyfree license to exercise all rights under the copyright claimed herein for Governmental purposes. All other rights are reserved by the copyright owner.
}

errors that occur in aircraft pressure-measurement devices. ${ }^{1}$

The investigation reported in this paper was prompted by the occurrence of large errors in the measurement of static pressure during lateral flight-test maneuvers of a V/STOL research aircraft (VSRA). Observation of the data revealed a strong correlation between the pressure and sideslip-angle measurement records. An algorithm based on potential flow over a cylinder was developed to correct the pressure record for sideslip-induced errors. The algorithm was modified to account for separation of the laminar boundary layer. In order to properly apply the correction algorithm, it was necessary to estimate and correct the lag in the pressure system. The method developed for estimating pressure lag is based on the coupling of sideslip activity into the static ports and can be used as a standard flight-test procedure.

The paper proceeds as follows: First, the cause and effect of the pressure errors observed during flight test are indicated and then the correction algorithm is derived. The flight-data procedures for applying the pressure correction and estimating the separation angle and pressure lag are described, and results are shown for a typical lateral maneuver. Finally, the implications of the method are discussed in the concluding remarks.

\section{Problem Statement}

The VSRA is equipped with the industry-standard flight-test boom shown in Fig. 1. The boom houses the pitot static sensors for measurement of total and static pressure, as well as the flow-angle sensors for measurement of angles of attack (alpha vane) and sideslip (beta vane). The static pressure is sensed at two closely spaced ports located symmetrically on the top and bottom of the cylindrical tube about four diameters aft of the pitot head. The tube diameter at the static ports is 0.62 inch, a value for which, from a cross-flow standpoint, the Reynold's number will remain subcritical, even at large yaw angles and high airspeed. ${ }^{2}$ Any separation of the boundary layer from the cylinder near the static ports will therefore be laminar in nature. 
The beta-vane and static-pressure records shown in Fig. 2 are observed to be strongly correlated. The effect of sideslip-induced pressure error is evident in the record of calculated pressure altitude, where it is seen that a sideslip of $10^{\circ}$ causes an altitude variation of about 100 feet. It should be mentioned here that the nose-boom manufacturer offers a test program to provide individual calibration curves of pitot and static pressure as a function of Mach number, angle of attack, and sideslip over a customer-specified flight regime. It was decided, however, to try to express the static-pressure errors on a yawed cylinder analytically by using a potential-flow approach, suitably modified to include boundary-layer separation. ${ }^{3}$ The resulting algorithm could then be used to correct the static-pressure record. This approach to the problem is described in the sections that follow.

\section{Correction Algorithm}

The effect of a yawed airflow over the cylindrical nose boom will be modeled by assuming that the flow is frictionless, and that the cross flow and axial flow can be considered separately. It is also assumed that the nose boom is aligned with the longitudinal body axis of the aircraft. The plane containing the static ports is shown in Fig. 3(a), where, to simplify the analysis, only one upper and one lower port are indicated. The potential flow solution for the cross-flow component of tangential velocity at the lower static port is given by 4

$$
V_{c}=2\left(v^{2}+w^{2}\right)^{1 / 2} \sin \theta
$$

where $\mathrm{v}, \mathrm{w}$ are the components of the free-stream velocity in the plane perpendicular to the cylinder, and $\theta$ is the angle between the line joining the stagnation points and the line joining the static ports. Now, if $u$ is the axial component of free-stream velocity, the magnitude of the tangential velocity at the lower port is

$$
V=\left[u^{2}+4\left(v^{2}+w^{2}\right) \sin ^{2} \theta\right]^{1 / 2}
$$

The error in static pressure at the lower port, expressed in coefficient form, is given by

$$
\Delta C_{p}=\left(p-p_{\infty}\right) /\left(\rho V_{t}^{2} / 2\right)
$$

where $p$ is the pressure sensed at the port, $p_{\infty}$ is the freestream static pressure, $p$ is the density, and $V_{t}$ is the magnitude of the free-stream velocity. Note that

$$
V_{t}=\left(u^{2}+v^{2}+w^{2}\right)^{1 / 2}
$$

The use of Bernoulli's equation ${ }^{4}$ to express the relation between pressure and velocity allows Eq. (3) to be written as

$$
\Delta C_{p}=1-v^{2} / V_{t}^{2}
$$

Note that the flow-angle sensors mounted on the nose boom near the static ports have the relations

$$
\tan \beta=v / u ; \quad \tan \alpha=w / u
$$

where $\beta$ is the angle sensed by the beta vane, and $\alpha$ is the angle sensed by the alpha vane. It can be seen from Fig. 3(a) that

$$
\tan \theta=v / w=\tan \beta / \tan \alpha
$$

The expressions of Eqs. (2), (4), and (6) can be used to write Eq. (5) as

$$
\begin{aligned}
\Delta C_{p} & =K\left(1-4 \sin ^{2} \theta\right) \\
K & =\left(\tan ^{2} \beta+\tan ^{2} \alpha\right) /\left(1+\tan ^{2} \beta+\tan ^{2} \alpha\right)
\end{aligned}
$$

Were the flow over the cylinder truly inviscid, the pressure sensed at the upper port would be the same as that at the lower port. Empirical studies have shown, however, that boundary-layer separation does occur (as illustrated in Fig. 3(b)), so that the upper and lower ports sense different pressures. For example, a port in the wake of a separated boundary layer would contribute an error of

$$
\Delta C_{p}=K\left(1-4 \sin ^{2} \theta_{s}\right)
$$

where $\theta_{8}$ is the angle at which separation occurs. Now, if it is assumed that $\theta_{\mathrm{s}}<90^{\circ}$, then for $\theta<\theta_{\mathrm{s}}$, only one port is in the wake of separated flow. For this case the average of the pressure errors is given by

$$
\Delta C_{p}=K\left[1-2\left(\sin ^{2} \theta+\sin ^{2} \theta_{s}\right)\right]
$$

It follows that for $\theta>\theta_{s}$, both ports are in the wake of separated flow, so that the average pressure error is given by Eq. (9). The application of the algorithm given by Eqs. (9) and (10) for correction of the static pressure record is described next.

\section{Application}

The pressure-correction algorithm derived in the previous section is a function of the alpha and beta vane angles, and the flow-separation angle. A block diagram outlining the application of the algorithm is shown in Fig. 4. Note that any lag in the VSRA pressure system 
must be accounted for, since the sideslip error is induced at the static-port location, while the pressure transducer is located 25 feet aft of the static ports, with the usual preumatic tube interconnection. Before making the lag correction, however, the separation angle must be estimated, and, if the pressure system lag is not known, it also must be estimated from the data.

The procedure for estimating the separation angle is simple: The angle $\theta_{\mathrm{S}}$ is varied until the "best" correction of the low-frequency errors is obtained. For the VSRA nose boom, a value of $\theta_{s}=45^{\circ}$ produced the best correction. It is interesting to note that for $\theta_{3}=45^{\circ}$, Eq. ( 7 ) can be used with Eq. (10) to obtain the expression

$$
\Delta C_{p}=-2 \tan ^{2} \beta /\left(1+\tan ^{2} \beta+\tan ^{2} \alpha\right)
$$

where it is seen that no correction is necessary for zero sidestip. Hence, it would appear that the boom design is optimized for longitudinal maneuvers. This seems to be the logical choice, since these maneuvers are the most important in flight-test performance studies.

The procedure for estimating the pressure system lag is as follows: A first-order curve fit to the raw pressure coefficient produced residuals that enhanced the sideslipinduced errors. The residuals were then correlated with the correction coefficient $\Delta C_{p}$ (with $\theta_{3}=45^{\circ}$ ). The corresponding correlation curve is shown in Fig. 5 . The pressure-system lag is seen from that curve to be about $0.35 \mathrm{sec}$. A lag model ${ }^{1}$ consisting of a pure (acoustic) delay of $0.1 \mathrm{sec}$ in tandem with a simple first-order lag of $0.35 \mathrm{sec}$ removed the lag from the correlation curve and realized a suitable attenuation of high-frequency errors when the sideslip correction was applied.

The sideslip and corrected static-pressure and pressure-altitude records are shown in Fig. 6. The raw and corrected pressure records were also used to generate records of pressure altitude (corrected for temperature ${ }^{5}$ ) that are shown in Fig. 7 with the inertial (radar-derived) altitude for comparison. There the sideslip-induced errors are seen to have been reduced by more than a factor of five. Similar results have been obtained with other maneuvers that have significant lateral excitation. It should be noted that the coupling that exists between sideslip and static pressure might be used in a standard flight-test procedure for calibration of pressure lag. It would be desirable, however, to perform a ground test of the pressure system to validate the lag model.

\section{v. Concluding Remarks}

What appeared to be a serious cross-coupled staticpressure measurement problem during lateral maneuvers has been solved by applying an algorithm based on potential-flow theory. The algorithm accounts for viscous effects that cause separation of the nose-boom boundary layer. Furthermore, the coupling of sideslip activity into the static-pressure port makes it possible to perform a flight-data pressure-lag calibration that could be included as a standard flight-test procedure for research aircraft.

\section{References}

[1] Gracey, W.: "Measurement of Aircraft Speed and Altitude," NASA RP-1046, May 1980.

[2] Bursnall, W. J.; Loftin, L. K.: "Experimental Investigation of the Pressure Distribution About a Yawed Cylinder in the Critical Reynold's Number Range," NACA TN-2463, September 1951.

[3] Sears, W. R.: "The Boundary Layer of Yawed Cylinders," J. Aeronaut. Sci., Vol. 15, No. 1, January 1948.

[4] White, F. M.: Fluid Mechanics, McGraw-Hill, New York, 1979. Ch. 7.

[5] Dunlap, E. W.; Porter, M. B.: "Theory of the Measurement and Standardization of In-Flight Performance of Aircraft," AFFTC, Edwards, CA, Rept. FTC-TD-71-1. April 1971. 


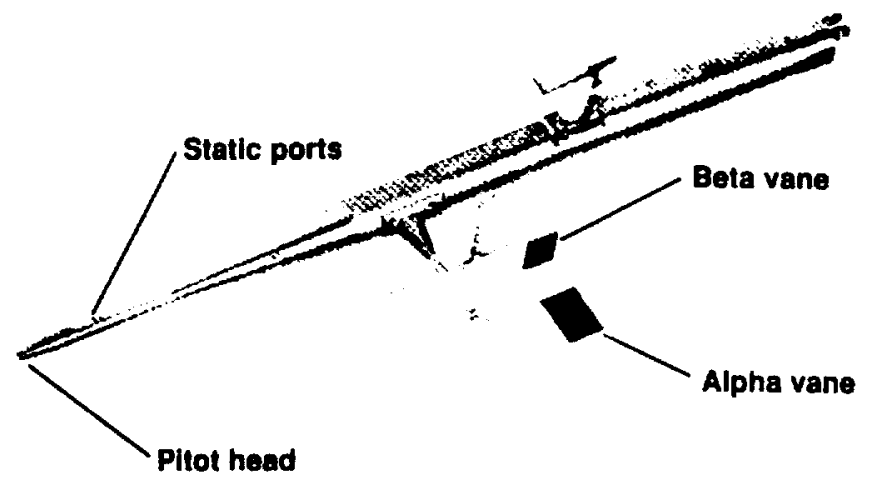

Fig. 1 VSRA air-data flight-test nose boom.
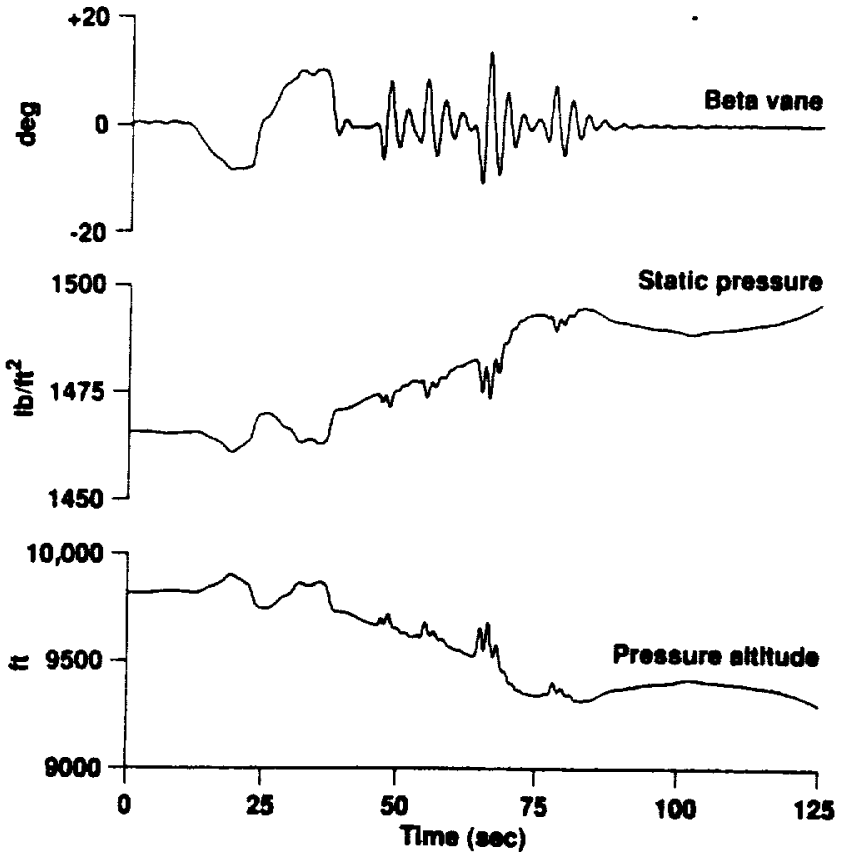

Fig. 2 Effect of sideslip on static pressure and pressure altitude.

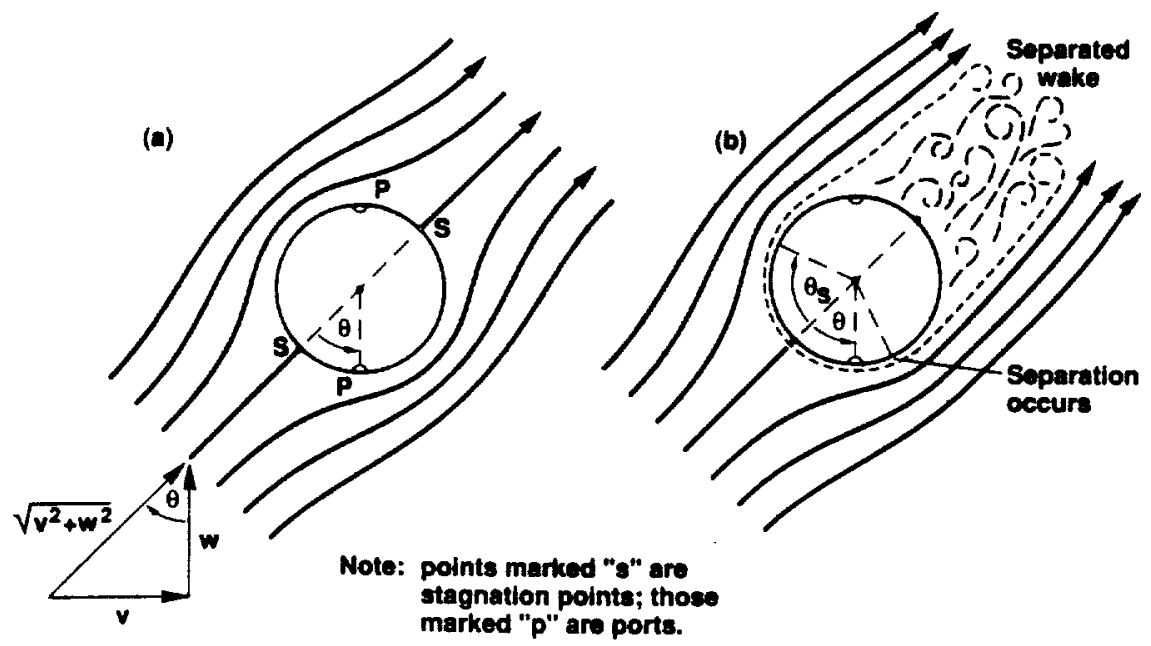

Fig. 3 Flow over nose-boom cross section at the static ports: (a) potential flow; (b) viscous flow. 


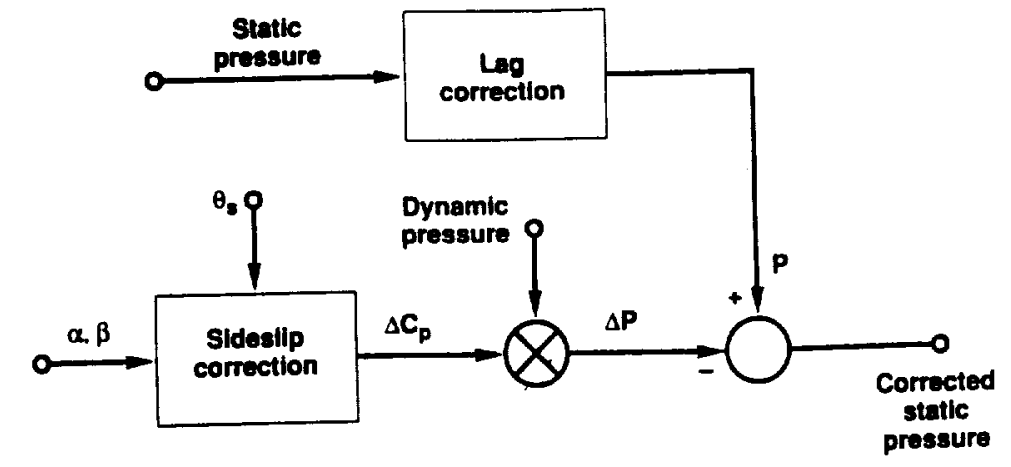

$$
\begin{aligned}
& \theta \leq \theta_{8}: \Delta C_{p}=K\left[1-2\left(\sin ^{2} \theta+\sin ^{2} \theta_{g}\right)\right] \\
& \theta>\theta_{g}: \Delta C_{p}=K\left(1-4 \sin ^{2} \theta_{s}\right) \\
& \theta=\arctan (\tan \beta / \tan \alpha) ; K=\left(\tan ^{2} \beta+\tan ^{2} \alpha\right) /\left(1+\tan ^{2} \beta+\tan ^{2} \alpha\right)
\end{aligned}
$$

Fig. 4 Correction of static pressure for lag and sideslip-induced errors.

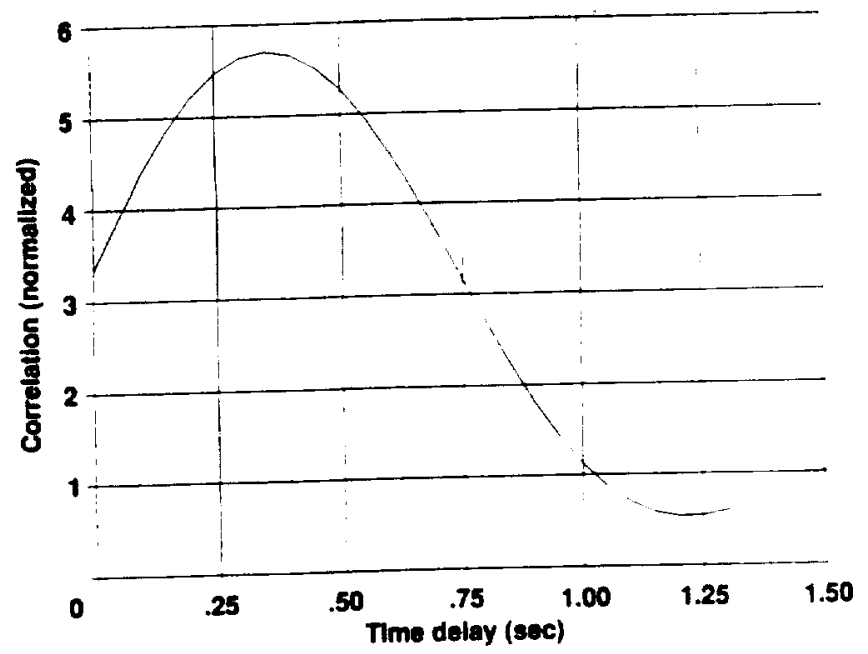

Fig. 5 Correlation of $\Delta \mathrm{Cp}$ with raw pressure coefficient for $\theta_{3}=45^{\circ}$.

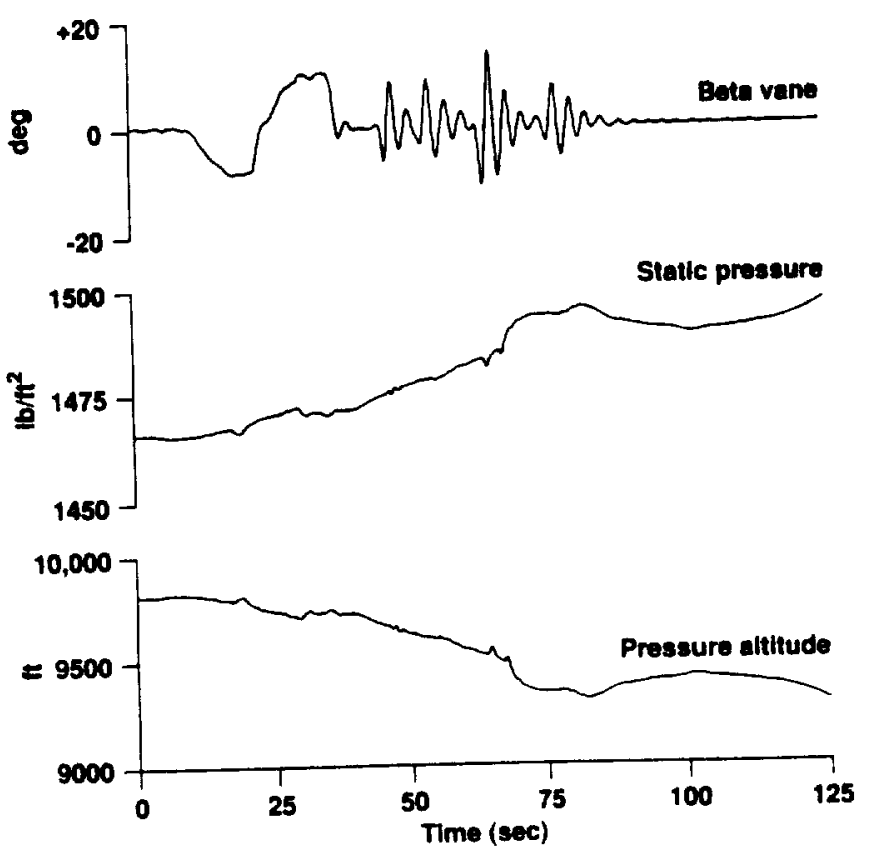

Fig. 6 Sideslip with corrected static-pressure and pressurealtitude records. 


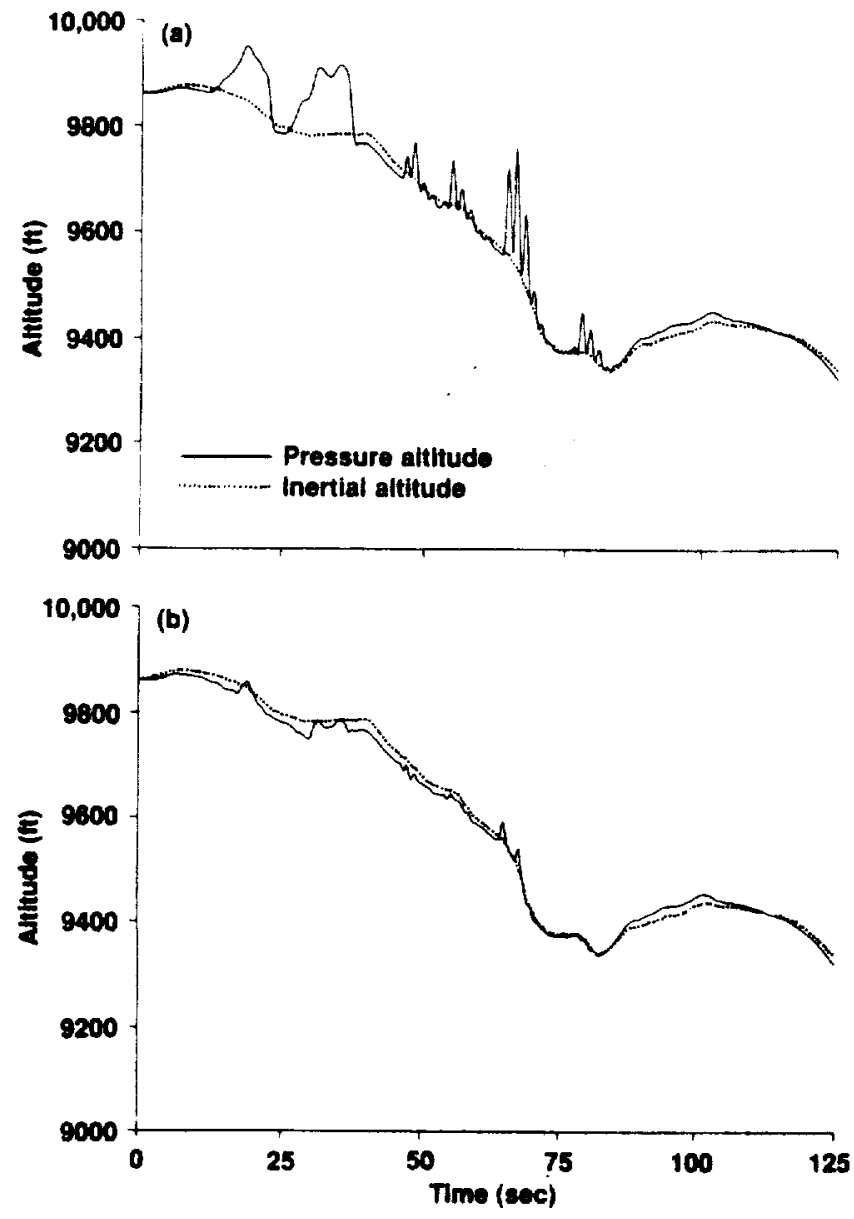

Fig. 7 Pressure altitude plotted with inertial altitude: (a) uncorrected; (b) corrected. 


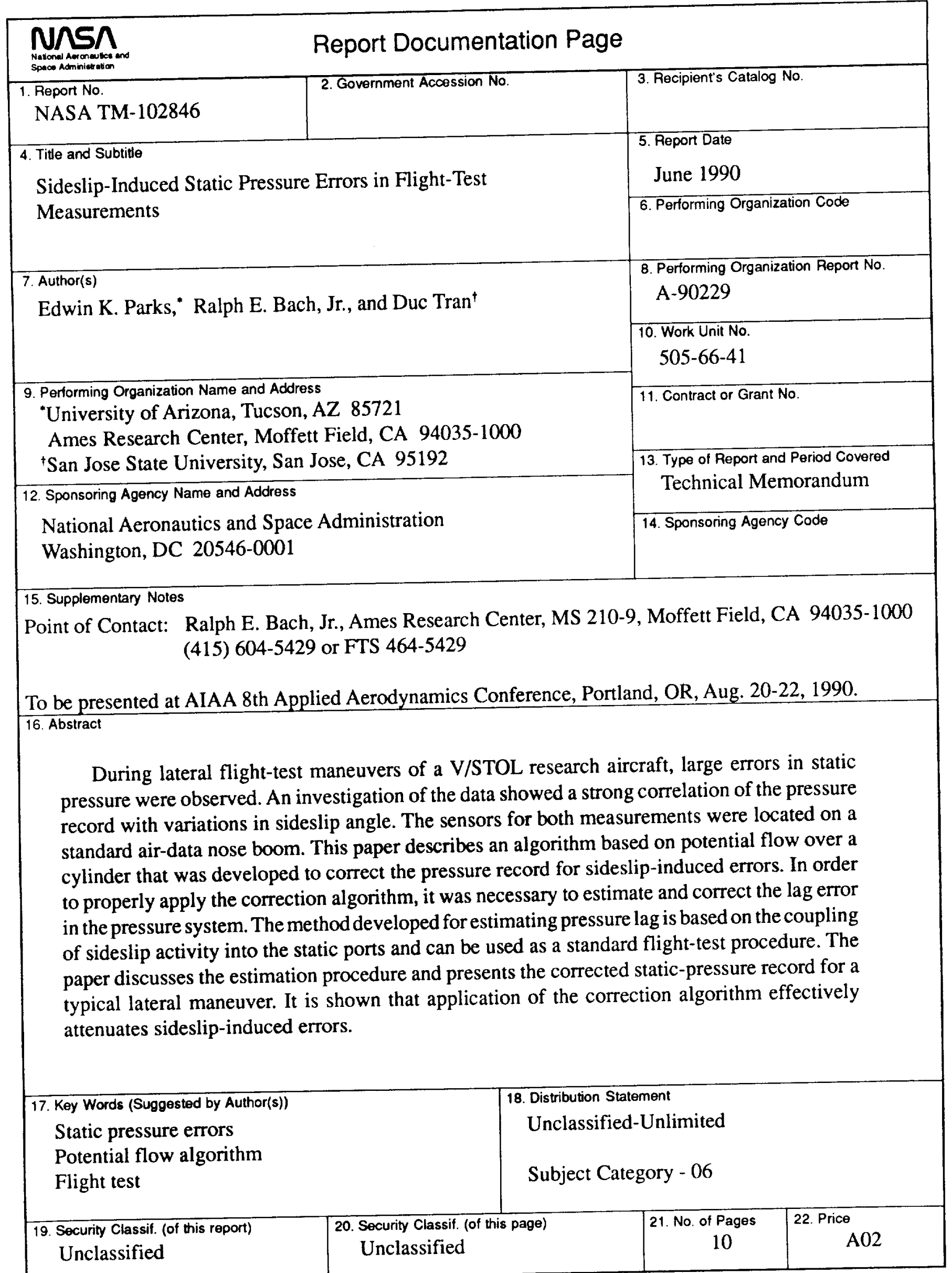


